\title{
Human leukocyte antigen class II-based immune risk model for recurrence evaluation in stage I-III small cell lung cancer
}

\author{
Peixin Chen (10 , ${ }^{1,2}$ Lishu Zhao (1) , , ${ }^{1,3}$ Hao Wang, ${ }^{1,2}$ Liping Zhang, ${ }^{4}$ Wei Zhang, ${ }^{4}$ \\ Jun Zhu, ${ }^{1,2}$ Jia Yu, ${ }^{1}$ Sha Zhao, ${ }^{1}$ Wei Li, ${ }^{1}$ Chenglong Sun, ${ }^{1,5}$ Chunyan Wu, ${ }^{4}$ \\ Yayi He (D) , ${ }^{1,2}$ Caicun Zhou (1D) ${ }^{1,2}$
}

To cite: Chen P, Zhao L, Wang $\mathrm{H}$, et al. Human leukocyte antigen class II-based immune risk model for recurrence evaluation in stage I-III small cell lung cancer. Journal for ImmunoTherapy of Cancer 2021;0:e002554. doi:10.1136/ jitc-2021-002554

- Additional supplemental material is published online only. To view, please visit the journal online (http://dx.doi.org/10. 1136/jitc-2021-002554).

PC and LZ contributed equally.

PXC and LSZ are joint first authors.

Accepted 17 June 2021

Check for updates

(C) Author(s) (or their employer(s)) 2021. Re-use permitted under CC BY. Published by BMJ.

For numbered affiliations see end of article.

Correspondence to Dr Yayi He; 2250601@qq.com

\section{ABSTRACT}

Background Immunotherapy has revolutionized therapeutic patterns of small cell lung cancer (SCLC). Human leukocyte antigen class II (HLA class II) is related to antitumor immunity. However, the implications of HLA class II in SCLC remain incompletely understood.

Materials and methods We investigated the expression patterns of HLA class II on tumor cells and tumorinfiltrating lymphocytes (TILs) by immunohistochemistry staining and its association with clinical parameters, immune markers, and recurrence-free survival (RFS) in 102 patients with stage I-III SCLC with radical surgery. Additionally, an HLA class II-based immune risk model was established by least absolute shrinkage and selection operator regression. With bioinformatics methods, we investigated HLA class II-related enrichment pathways and immune infiltration landscape in SCLC.

Results HLA class II on tumor cells and TILs was positively expressed in $9(8.8 \%)$ and $45(44.1 \%)$ patients with SCLC, respectively. HLA class II on TILs was negatively associated with lymph node metastasis and positively correlated with programmed death-ligand 1 (PD-L1) on TILs $(p<0.001)$ and multiple immune markers (CD3, CD4, CD8, FOXP3; $p<0.001$ ). Lymph node metastasis (OR 0.314, $95 \% \mathrm{Cl} 0.118$ to $0.838, \mathrm{p}=0.021$ ) and PD-L1 on TILs (OR $3.233,95 \% \mathrm{Cl} 1.051$ to $9.95, \mathrm{p}=0.041$ ) were independent predictive factors of HLA class II on TILs. HLA class II positivity on TILs prompted a longer RFS (40.2 months, $95 \% \mathrm{Cl} 31.7$ to 48.7 vs 28.8 months, $95 \% \mathrm{Cl} 21.4$ to 36.3 , $\mathrm{p}=0.014)$. HLA class II on TILs, PD-L1 on TILs, CD4, and FOXP3 were enrolled in the immune risk model, which categorized patients into high-risk and low-risk groups and had better power for predicting the recurrence than tumor stage. Pathway enrichment analyses showed that patients with high HLA class II expression demonstrated signatures of transmembrane transportation, channel activity, and neuroactive ligand-receptor interaction. Highrisk SCLC patients had a higher proportion of T follicular helper cells $(p=0.034)$ and a lower proportion of activated memory CD4-positive T cells $(p=0.040)$ and resting dendritic cells $(p=0.045)$ versus low-risk patients. Conclusions HLA class II plays a crucial role in tumor immune microenvironment and recurrence prediction. This work demonstrates the prognostic and clinical values of HLA class II in patients with SCLC.

\section{BACKGROUND}

Worldwide, lung cancer has the highest mortality rate with small cell lung cancer (SCLC) making up $10 \%-15 \% .^{12}$ SCLC is known as an aggressive and recalcitrant disease in that it has fast doubling time, high growth fraction, early extensive dissemination, as well as no remarkable advances in therapeutic methods in the past three decades. ${ }^{23}$ SCLC can be classified into limitedstage SCLC (LS-SCLC) and extensive-stage SCLC (ES-SCLC), and the latter accounts for $60 \%-70 \%{ }^{4}$ ES-SCLC is principally treated with platinum-based chemotherapy with a median overall survival (OS) of approximately 9-12 months. ${ }^{45}$ Despite an up to $75 \%$ initial sensitivity to platinum-based chemotherapy, patients with ES-SCLC will inevitably develop resistance. ${ }^{4}$

Recently, immunotherapy has revolutionized cancer therapy due to promising antitumor activity and significantly improved OS. ${ }^{6}$ Notably, immunotherapy has shown therapy promise and has broken the situation of no superior treatment than chemotherapy in SCLC. ${ }^{78}$ IMpower133, a phase 3 clinical trial, demonstrated a better prognosis among patients who took administration of atezolizumab and chemotherapy versus those who received chemotherapy alone. ${ }^{7}$ Subsequent CASPIAN study obtained similar results. ${ }^{9}$ However, immunotherapy improves the survival of patients with SCLC by only 2 months. For patients with SCLC, little progress has been made in efficacious predictive biomarkers including programmed deathligand 1 (PD-L1). ${ }^{10}$ Therefore, identification of patients who may benefit from immunotherapy is urgently needed.

Human leukocyte antigen (HLA), also called major histocompatibility complex, 
exerts critical roles in the interaction between tumor cells and the human immune system. HLA class I is one of the canonical HLA molecules, and the role of HLA class I-specific CD8-positive $\mathrm{T}$ cell responses in antitumor immune response has been well established. ${ }^{11-13}$ Canonically coded by HLA-DP, DQ, and DR, HLA class II is highly restricted to professional antigen-presenting cells (APCs) and participates in exogenous antigen presentation to CD4+ T cells. ${ }^{1114}$ Nonetheless, HLA class II expression on tumor cells and immune cells can be induced by interferon (IFN)- $\gamma .{ }^{1516}$ Evidence showed HLA class II on tumor cells affected the tumor immunogenicity, tumor migration and invasion, cancer progression, immune response, and prognosis in numerous malignancies in vitro and in vivo. ${ }^{17-19}$ HLA class II on tumor-infiltrating lymphocytes (TILs) was related to antigen presentation, interactions with immune cells, intracellular signaling, and prognosis of patients with cancer. ${ }^{20}{ }^{21}$ Besides, HLA class II on TILs had a controversial correlation with tumor metastasis to lymph nodes in gastric cancer. ${ }^{22}$ Compared with nonsmall cell lung cancer (NSCLC), reduced expression of HLA class II on TILs in SCLC was considered as a form of tumor immune escape. ${ }^{21}$ However, the implications of HLA class II on TILs in tumor immune microenvironment and prognosis in SCLC are not completely understood.

Herein, this study aims to analyze expression patterns of HLA class II on tumor cells and TILs by immunohistochemical (IHC) staining, and its association with clinical and immune indicators and recurrence-free survival (RFS) in SCLC. Additionally, we established an HLA class II-based immune risk model by least absolute shrinkage and selection operator (LASSO) regression and investigated the enrichment pathways and immune infiltration landscape associated with HLA class II in SCLC by bioinformatics analysis.

\section{MATERIALS AND METHODS Patients}

Altogether, 102 patients with SCLC receiving radical resection in Shanghai Pulmonary Hospital between 2014 and 2018 were selected. Clinical features were gathered from electrical medical records. Lung cancer staging was determined by the seventh tumor, node, metastases staging system. ${ }^{23}{ }^{24}$ Surgically resected tumor samples were histologically reviewed. All patients signed informed consent.

\section{IHC staining}

All formalin-fixed paraffin-embedded tumor tissues were dewaxed with xylene, hydrated with gradient alcohol, and washed in distilled water. And then antigen retrieval was accomplished with the target recovery solution kit (DM828 or DM829, Dako). The detailed procedure was performed as described in our previous studies. ${ }^{2125}$ Experimental antibodies were rabbit antihuman HLA class II (HLA class II-DP, HLA class II-DQ, HLA class II-DR, CR3/43, Abcam), program death-1 (PD-1) (Golden bridge Zhongshan, Beijing ZM-0381), PD-L1 (E1L3N, CST \# 13684S), CD3 (Dako A0452), CD4 (Dako M7310), CD8 (Dako M7103), and FOXP3 (BioLegend 320101).

\section{Cut-off values of HLA class II and other immune markers}

The IHC staining results were independently reviewed by two pathological physicians (CW and LZhang). When the results of two independent pathologists differed, they discussed the decisions together to reach a consensus. The optimal cut-off values were determined based on survival analyses. ${ }^{25}{ }^{26} \mathrm{X}$-tile software ${ }^{27}$ (V3.6.1) was used to generate the cut-off values of immune parameters except for PD-L1 expression on tumor cells. The cut-off points for HLA class II staining positivity on cancer cells and TILs were $5 \%$ and $25 \%$, respectively (online supplemental figure 1A,B). The positive cut-off values of other immune markers including PD-1, PD-L1 on TILs, CD3, CD4, CD8, as well as FOXP3 were 1\%, 5\%, 40\%, 30\%, 30\%, and 10\%, respectively, as we demonstrated before. ${ }^{28}$ PD-L1 tumor proportion score $\geq 1 \%$ was considered positive.

\section{Establishment of immune risk model by LASSO regression}

LASSO regression was applied to build an immune risk model on the basis of HLA class II. LASSO regression obtained a more refined and stable model by constructing a penalty function, compressing some coefficients, and setting some insignificant coefficients equal to zero. LASSO regression retains the advantage of subset contraction and gets good results when processing data that have complex collinearity. We obtained the LASSO risk model using EmpowerStats (V3.0, Solutions, Boston, Massachusetts, USA, R V3.4.3) based on lambda (min), that is, the lambda whose error mean was the smallest. Then X-tile software (V3.6.1) was employed to determine the optimal cut-off value that separated patients into high-risk and low-risk subgroups. The discrimination of this model was evaluated by the area under the curve (AUC) of receiver operating characteristic.

\section{Clinical value of HLA class II and immune risk model in SCLC} We further assessed the clinical and predictive value of HLA class II and immune risk model by downloading SCLC dataset from cBioportal Database (https://www. cbioportal.org). Samples were enrolled if complete mRNA sequencing data and survival data of patients with SCLC were accessible.

\section{Validation of HLA class II expression in SCLC}

To further explore the expression levels of HLA class II gene and its subtypes, we downloaded RNA sequencing data of tumor tissues and normal tissues in patients with SCLC in the GEO database. And we respectively compared the expression status of HLA class II along with its 11 subtypes (HLA-DRA, HLA-DRB1, HLA-DRB4, HLADRB6, HLA-DPA1, HLA-DPA2, HLA-DPB1, HLA-DPB2, HLA-DQA1, HLA-DQB1, and HLA-DQB2) in tumor tissues versus normal tissues. The average expression levels of 11 subtypes constituted the total expression level 
of HLA class II whose best cut-off value was determined by significantly separated survival curves.

\section{Gene ontology terms and KEGG pathway enrichment analysis}

Gene ontology (GO) and Kyoto Encyclopedia of Genes and Genomes (KEGG) enrichment analyses were performed with R (R V.3.6.3) on differentially expressed genes between high versus low HLA class II expression groups to investigate the discrepant biological pathways associated with HLA class II. ${ }^{29}$ GO terms consisted of three categories: molecular function (MF), cellular component (CC), and biological process (BP). Differentially expressed genes were defined at $\mathrm{p}<0.05$.

\section{Immune infiltration analysis in SCLC by CIBERSORT}

We used a public website tool CIBERSORT (https:// cibersort.stanford.edu) that provided mRNA expression profiles of 22 immune cells, that is, LM22, to clarify the landscape of immune infiltration in SCLC patients with high-risk versus low-risk. ${ }^{30}$ The immune risk was decided by RNA expression levels of HLA class II gene, CD4, FOXP3, and CD274 encoding PD-L1. Eighty-one SCLC samples were classified into high and low-risk groups. Immune maps were depicted and correlation analyses of each immune cell were performed in both groups. Additionally, the proportion of each immune cell was compared between high-risk and low-risk groups.

\section{Statistical analyses}

Statistical description and analyses were carried out as described in our former studies. ${ }^{21}{ }^{25}$ The Pearson $\chi^{2}$ and Fisher exact test were applied to analyze the association of HLA class II with clinical and immune indexes. Spearman rank correlation was performed for the analysis of the linear correlation between HLA class II and other immune markers. Binary logistic regression was done to determine the independently influential factors of HLA class II. Odds ratios (OR) and 95\% confidence intervals (CI) of variables were calculated in the logistic regression models. Survival data were estimated by the Kaplan-Meier method and compared with the log-rank test. The hazard ratios (HR) and 95\% CI of variables were determined by Cox regression. Statistical analyses across the full text were performed on GraphPad Prism (V7.0; La Jolla, California, USA) and IBM SPSS Statistics (V22.0; IBM,Chicago, IL, USA). When the two-sided $p$ values were less than 0.05 , the differences were considered statistically significant.

\section{RESULTS}

\section{Patient characteristics}

A total of 102 SCLC patients receiving radical surgery were enrolled in this study, among which $18(17.6 \%)$ were women, $23(22.5 \%)$ were aged $\geq 70$ years, and 58 (56.9\%) were never-smokers, as we showed previously. ${ }^{28}$ Among 102 patients with SCLC, 60 (58.8\%) presented with stage I or II, and others with stage III. Sixty-six (64.7\%) patients received adjuvant chemotherapy and 27 patients (26.5\%) accepted adjuvant radiotherapy.

\section{HLA class II and its correlation with clinical and immune parameters}

HLA class II was positively expressed on cancer cells in $9(8.8 \%)$ and on TILs in $45(44.1 \%)$ patients, respectively. Online supplemental table 1 listed their detailed expression status. No significant correlations were found between HLA class II on tumor cells and all clinical parameters including age, gender, smoking, T stage, $\mathrm{N}$ stage, distant metastasis, tumor stage, and chemotherapy. However, HLA class II on TILs was negatively associated with lymph node metastasis $(\mathrm{p}=0.002)$ (table 1$)$.

We then analyzed the relationship between HLA class II and immune parameters by $\chi^{2}$ test and spearman rank correlation (table 1 and online supplemental table 2). HLA class II on tumor cells was only related to PD-1 on TILs $(\mathrm{p}=0.028)$. However, the expression status of HLA class II on TILs was associated with many immune factors, such as PD-1 on TILs ( $\mathrm{p}=0.016)$, PD-L1 on TILs, CD3, CD4, CD8, and FOXP3 (all p<0.001, table 1). Subsequent spearman rank correlation test exhibited similar results. HLA class II expressed on TILs presented a significant linear correlation with multiple immune parameters including PD-1/PD-L1 on TILs, CD3, CD4, CD8, and FOXP3 (all $\mathrm{p}<0.001$, online supplemental table 2).

To further analyze possible clinical and immune factors associated with HLA class II, we performed logistic regression analysis. Rare expression of HLA class II on tumor cells in SCLC restrains further statistical analysis. Therefore, we could only include 14 variables and investigate the risk factors for positive expression of HLA class II on TILs. Significant variables in the univariate logistic regression (lymph node metastasis, PD-1 on TILs, PD-L1 on TILs, CD3, CD4, CD8, and FOXP3) were enrolled into the multivariate logistic regression model (online supplemental table 3). After adjusting other possible confounding factors, occurrence of lymph node metastasis (OR $0.294,95 \%$ CI 0.108 to $0.799, \mathrm{p}=0.016$ ) was an independent negative predictor of HLA class II on TILs. And patients with positive PD-L1 expression on TILs (OR $3.339,95 \%$ CI 1.071 to $10.404, p=0.038$ ) had a higher expression level of HLA class II on TILs compared with those negative (online supplemental table 3).

\section{Association of HLA class II and immune parameters with RFS}

A total of 102 patients with SCLC were included in the RFS analysis. Fifty-six patients $(54.9 \%)$ relapsed by the end of follow-up, and 49 (87.5\%) of 56 patients relapsed within 2 years after surgery.

We first analyzed the association of HLA class II expression with RFS in these SCLC patients with the KaplanMeier method and log-rank test. The status of HLA class II expression on tumor cells had no significant difference in RFS ( $p=0.083$ ) (figure 1A). Nonetheless, patients with positive HLA class II expression on TILs had a significantly longer RFS than those negative (40.2 months, 
Table 1 Association of HLA class II with clinical and immune parameters

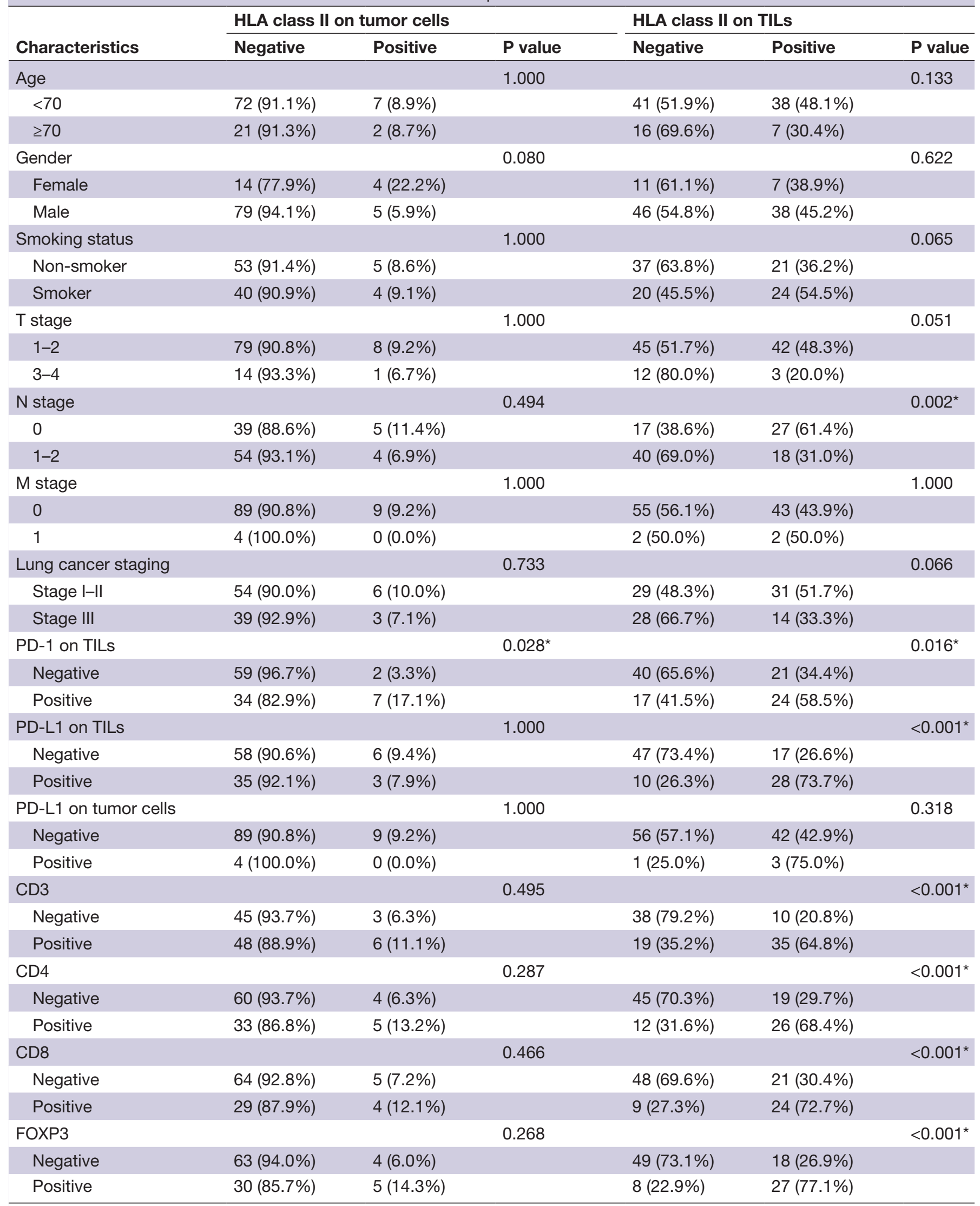

${ }^{*} \mathrm{P}<0.05$ indicates statistical significance.

HLA class II, human leukocyte antigen class II; PD-1, programmed death-1; PD-L1, programmed death-ligand 1; TILs, tumor infiltrating lymphocytes. 


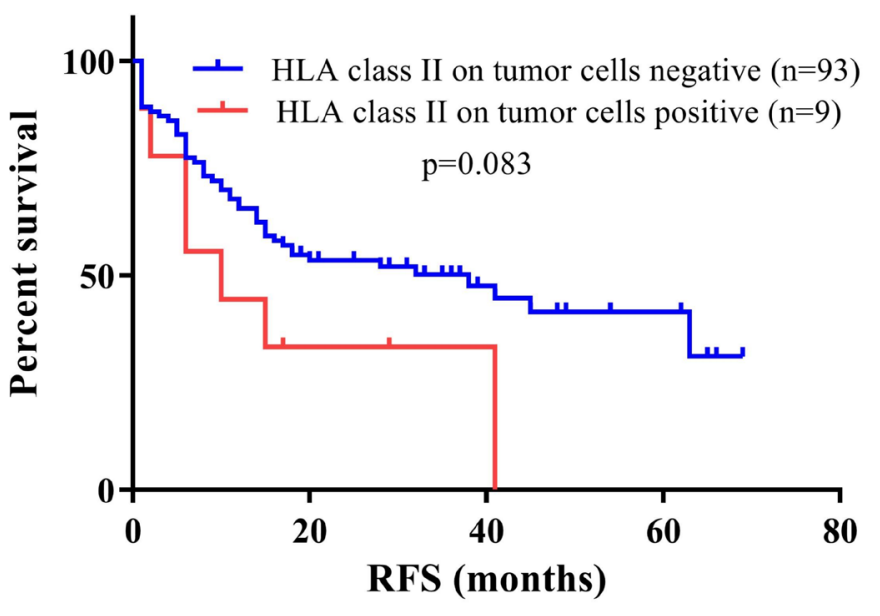

A) RFS by HLA class II status on tumor cells

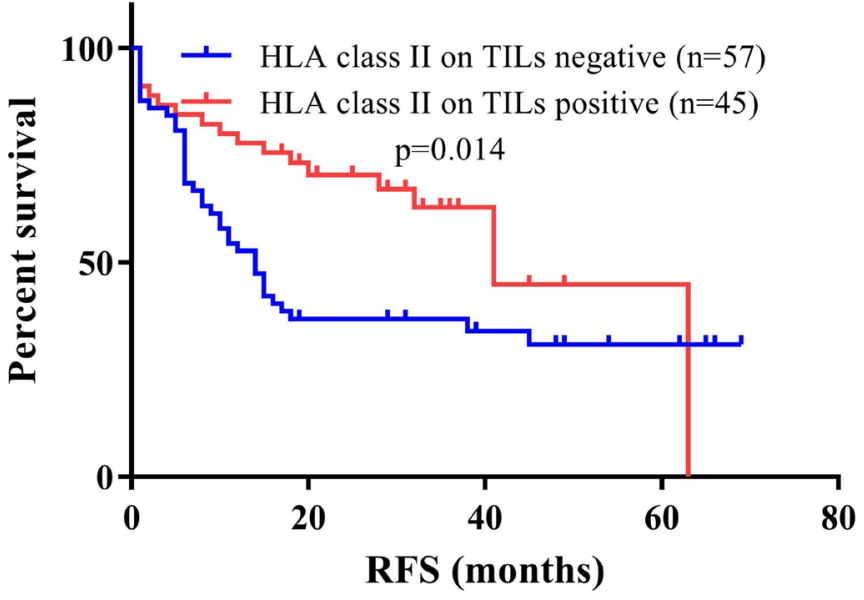

B) RFS by HLA class II status on TILs

Figure 1 Survival analysis by HLA class II on tumor cells and TILs. HLA class II, human leukocyte antigen class II; RFS, recurrence-free survival; TILs, tumor infiltrating lymphocytes.

95\% CI 31.7 to 48.7 months vs 28.8 months, $95 \%$ CI 21.4 to 36.3 months, $p=0.014$; figure $1 \mathrm{~B}$ ). The median RFS for patients with positive and negative HLA class II expression on TILs was 41 months (95\% CI 29.1 to 53.0) and 14 months (95\% CI 9.9 to 18.1 ), respectively.

We then enrolled HLA class II and other immune factors in the univariate Cox regression model to select possible prognostic indicators. RFS was related to the status of HLA class II expressed on TILs (HR 0.507, $95 \%$ CI 0.288 to $0.893, p=0.019$ ) rather than tumor cells (table 2). As our previous studies have shown, ${ }^{28}$ RFS was also correlated with PD-L1 on TILs, and CD3, CD4, CD8, and FOXP3 on immune cells but not with PD-1 on TILs and PD-L1 on tumor cells (table 2).

\section{Establishment of HLA class II-based immune risk model by LASSO regression}

The results of the univariate Cox regression indicated that six immune indicators (HLA class II on TILs, PD-L1 on TILs, CD3, CD4, CD8, and FOXP3) were significantly associated with RFS in patients with SCLC. Given that spearman's correlation analysis revealed that HLA class II on TILs had a modest linear correlation with other five immune factors, the traditional multivariate Cox regression is not applicable. Under this circumstance, we constructed an immune risk model by LASSO regression to comprehensively analyze the effects of immune indicators on the prognosis. After performing LASSO regression, we obtained two immune risk models. One is based on lambda.min corresponding to the minimum mean error and the second is on lambda.1se, that is, the maximum lambda corresponds to the minimum mean error within one standard deviation (figure 2A). Figure 2B showed the corresponding relationship between coefficients of each variable and the lambda. Based on the lambda.min (0.0279), we obtained the optimal risk model with an AUC of 0.709, which was superior to single immune markers and even tumor stage (AUC, 0.655 ) in terms of prediction efficiency (figure 2C). We

\begin{tabular}{|c|c|c|c|}
\hline Variables & HR & $95 \% \mathrm{Cl}$ & $P$ value \\
\hline HLA class II on TILs (negative vs positive) & 0.507 & 0.288 to 0.893 & $0.019^{*}$ \\
\hline HLA class II on tumor cells (negative vs positive) & 1.964 & 0.885 to 4.355 & 0.097 \\
\hline PD-1 on TILs (negative vs positive) ${ }^{28}$ & 0.602 & 0.342 to 1.059 & 0.078 \\
\hline PD-L1 on TILs (negative vs positive) ${ }^{28}$ & 0.417 & 0.223 to 0.779 & $0.006^{*}$ \\
\hline PD-L1 on tumor cells (negative vs positive) & 0.815 & 0.198 to 3.347 & 0.776 \\
\hline FOXP3 (negative vs positive) ${ }^{28}$ & 0.376 & 0.194 to 0.730 & $0.004^{*}$ \\
\hline CD3 (negative vs positive) ${ }^{28}$ & 0.480 & 0.281 to 0.820 & $0.007^{*}$ \\
\hline CD4 (negative vs positive) $)^{28}$ & 0.450 & 0.245 to 0.825 & $0.010^{*}$ \\
\hline CD8 (negative vs positive) ${ }^{28}$ & 0.400 & 0.206 to 0.776 & $0.007^{\star}$ \\
\hline
\end{tabular}

${ }^{*} \mathrm{P}<0.05$ indicates statistical significance.

HLA class II, human leukocyte antigen class II; PD-1, programmed death-1; PD-L1, programmed death-ligand 1; TILs, tumor infiltrating lymphocytes. 


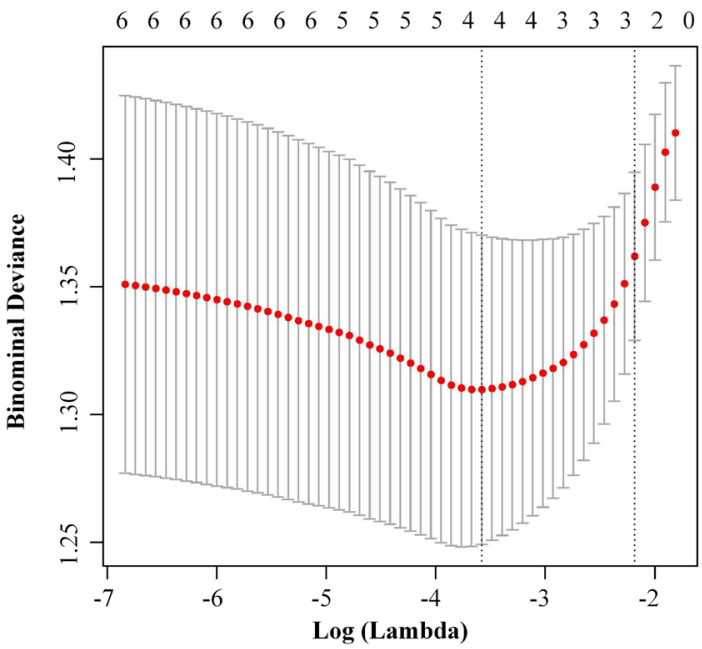

A) Lambda $(\lambda)$ selection in the LASSO regression

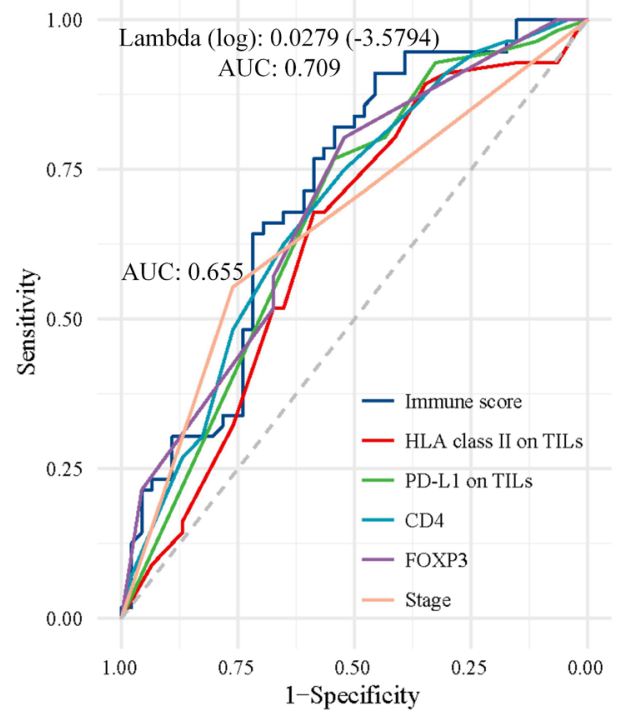

C) ROC curves and AUC values

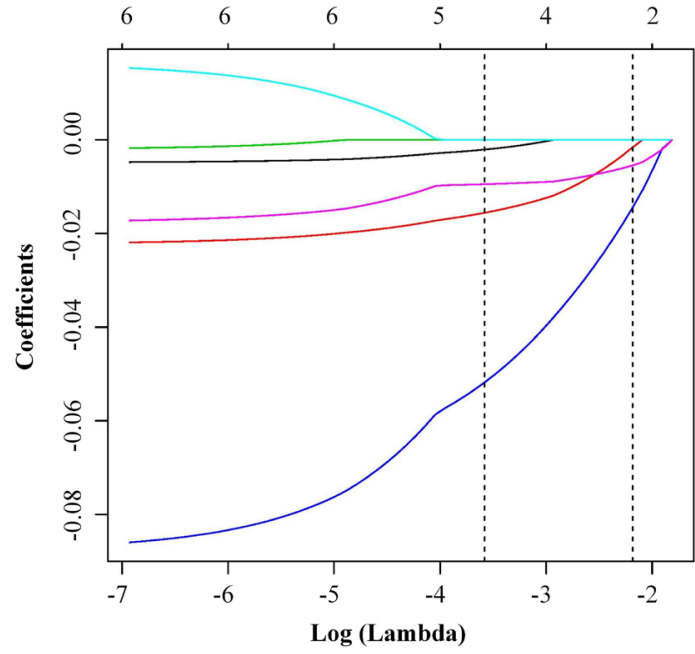

B) Coefficient profiles of the features against the $\log (\lambda)$

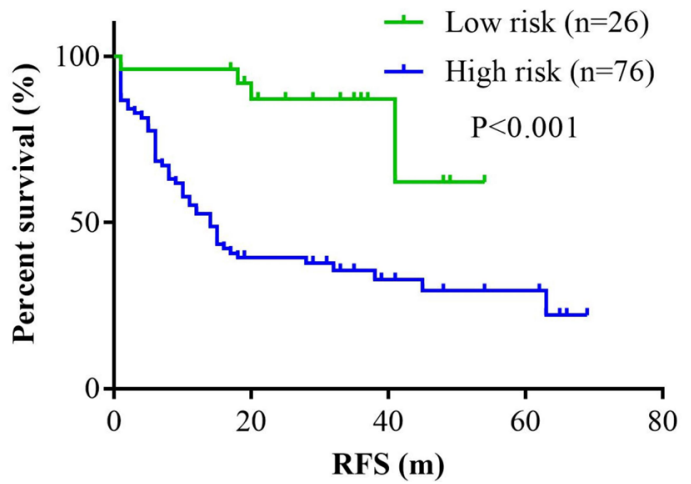

D) Survival analysis according to the risk score

Figure 2 Establishment of immune risk model in SCLC. (A) The lambda ( $\lambda$ ) selection process in the LASSO regression. Two immune risk models were obtained based on lambda.min (left dotted line) and lambda.1se (right dotted line). (B) LASSO coefficient profiles of each variable against the $\log (\lambda)$. The optimal immune risk model was achieved based on the lambda.min (left dotted line, 0.0279), and it enrolled four variables: HLA class II on TILs, PD-L1 on TILs, FOXP3, and CD4. (C) ROC curves and AUC values of immune risk model, single immune factors and tumor stage. The AUC value of immune risk score (0.709) exceeded that of tumor stage $(0.655)$ and single immune factors (all $<0.70$ ). (D) Survival analysis of immune risk score. AUC, area under the curve; HLA class II, human leukocyte antigen class II; LASSO, least absolute shrinkage and selection operator; PD-L1, programmed death-ligand 1; ROC, receiver operating characteristic; SCLC, small cell lung cancer; TILs, tumorinfiltrating lymphocytes.

eliminated less important factors CD3 and CD8 due to their coefficients were zero. The final LASSO formula was as follows: immune score $=-(0.00206 \times$ HLA class II on TILs) $-(0.01558 \times$ PD-L1 on TILs $)-(0.05179 \times$ FOXP3 $)-$ $(0.00947 \times \mathrm{CD} 4)$. Then we applied X-tile to determine the optimal cut-off value that separated patients into high-risk and low-risk groups. Survival analysis demonstrated that RFS in high-risk and low-risk patients with SCLC significantly differed $(\mathrm{p}<0.001)$ (figure 2D).

\section{Clinical value of HLA class II and immune risk model in SCLC} We further explored the clinical prognostic value of HLA class II and immune risk model in SCLC from the transcriptional level. In the public dataset, ${ }^{31} 77$ SCLC patients with survival data were enrolled and divided into high-expression and low-expression groups based on the expression level of HLA class II. OS curves of two groups were significantly separated $(\mathrm{p}=0.016$; online supplemental figure 2A). Additionally, patients with SCLC were also categorized into high-risk and low-risk groups based on RNA expression levels of four genes (HLA class II, CD274 encoding PD-L1, FOXP3, CD4). The KaplanMeier curve showed that high-risk patients presented a significantly poorer OS $(\mathrm{p}=0.0013)$ (online supplemental figure $2 \mathrm{~B}$ ). 


\section{Validation of HLA class II expression in SCLC}

To further explore the expression levels of HLA class II gene and its subtypes in SCLC, we downloaded RNA sequencing data of tumor and normal tissues in patients with SCLC in the GEO database. In the GSE43346 dataset, SCLC patients had a lower HLA class II expression level in comparison with normal tissues $(\mathrm{p}=0.043)$ (online supplemental figure 3A). For the HLA class II subtypes, we demonstrated that HLA-DRA $(\mathrm{p}=0.046)$, HLA-DRB1 $(\mathrm{p}=0.010)$, HLA-DRB4 $(\mathrm{p}=0.017)$, HLA-DRB6 $(\mathrm{p}=0.004)$, HLA-DPB2 $(\mathrm{p}=0.0005)$, and HLA-DQB1 $(\mathrm{p}=0.007)$ of tumor tissues were lower than that of normal tissues in SCLC patients, while HLA-DPA1, HLA-DPA2, HLADPB1, HLA-DQA1, and HLA-DQB2 were all not $(\mathrm{p}>0.05)$ (online supplemental figure 3B,C).

\section{GO and KEGG enrichment analysis of HLA class II expression}

To further investigate the biological pathways associated with HLA class II expression, we accomplished GO and KEGG enrichment analysis in SCLC patients in the GSE43346 dataset. As for GO terms enrichment, we exhibited respective top 10 enriched GO terms in MF, CC,and BP. HLA class II expression-related genes were prominently involved in presynapse (CC), synaptic membrane (CC), metal ion transmembrane transporter activity (MF), channel activity (MF), and passive transmembrane transporter activity (MF) (figure 3A). Besides, KEGG pathway analysis revealed that targeted genes were mainly clustered in the neuroactive ligand-receptor interaction signaling pathway (figure 3B).

\section{Landscape of immune infiltration in high-risk versus low-risk groups}

To further investigate the association of immune infiltration with immune risk score, we used CIBERSORT to assess the distribution and composition of 22 immune cells among patients with SCLC. Based on mRNA expression levels of HLA class II gene, CD4, FOXP3, and CD274 encoding

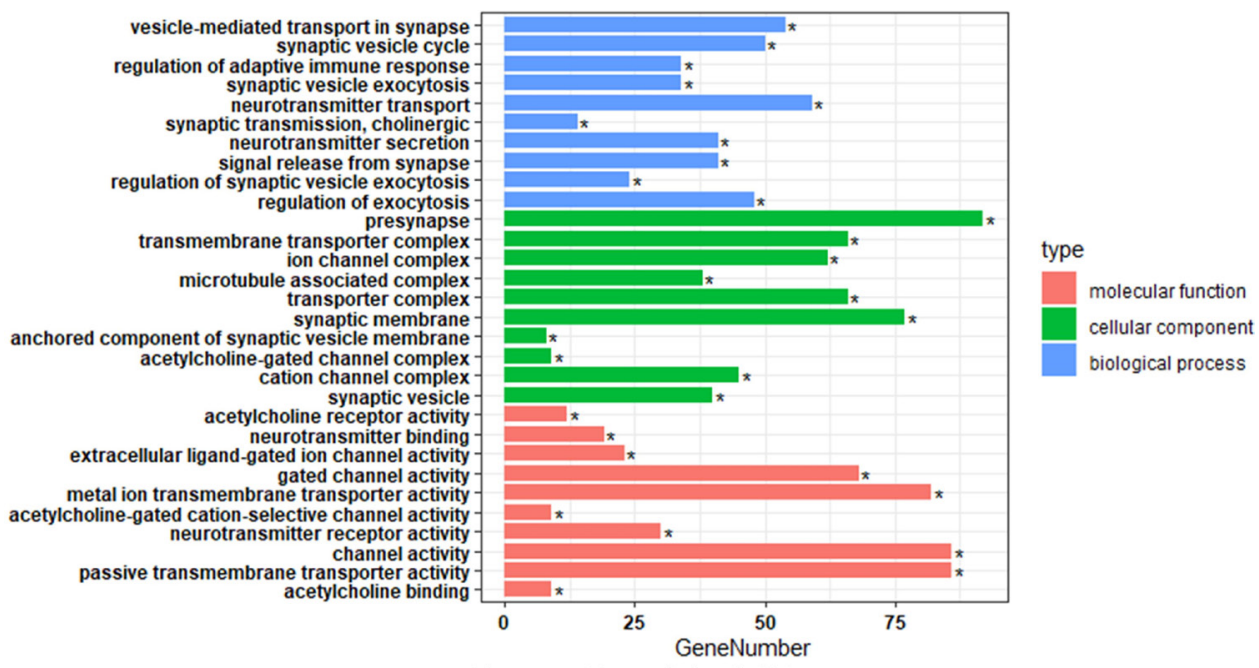

A) Top 10 enriched GO terms

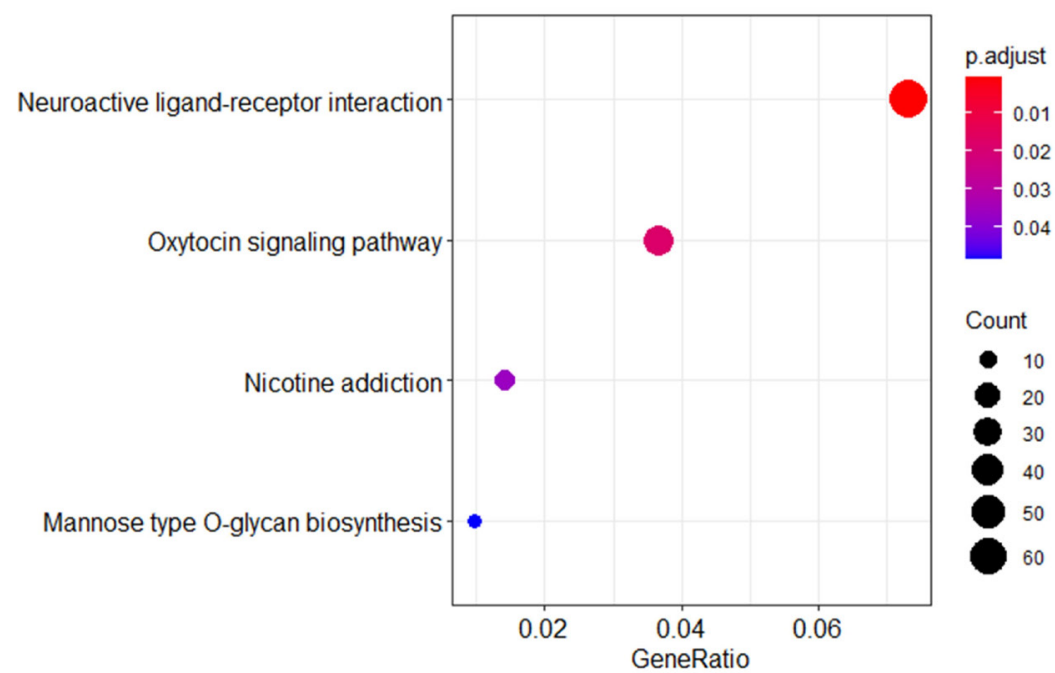

B) KEGG pathway enrichment analysis

Figure $3 \mathrm{GO}$ and KEGG enrichment analysis of HLA class II expression in SCLC. (A) Top 10 enriched GO terms in molecular function, cellular components, and biological processes. (B) KEGG pathway enrichment analysis. GO, gene ontology; HLA class II, human leukocyte antigen class II; KEGG, Kyoto Encyclopedia of Genes and Genomes; SCLC, small cell lung cancer. 


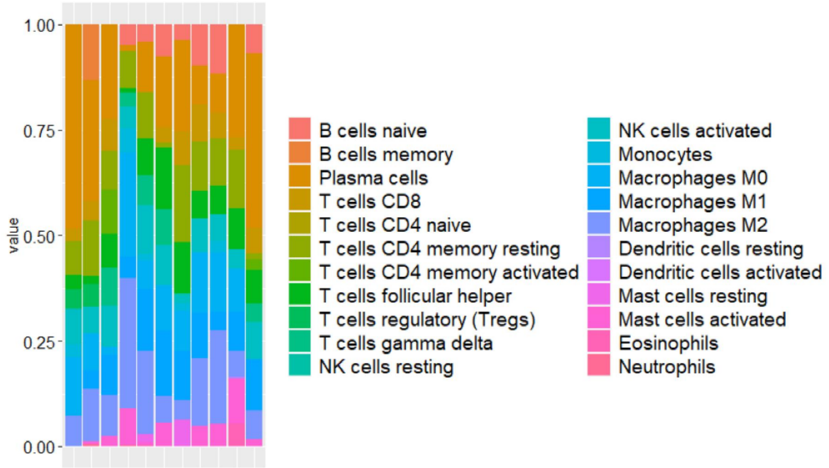

A) The proportion of 22 immune cells in high-risk patients with SCLC

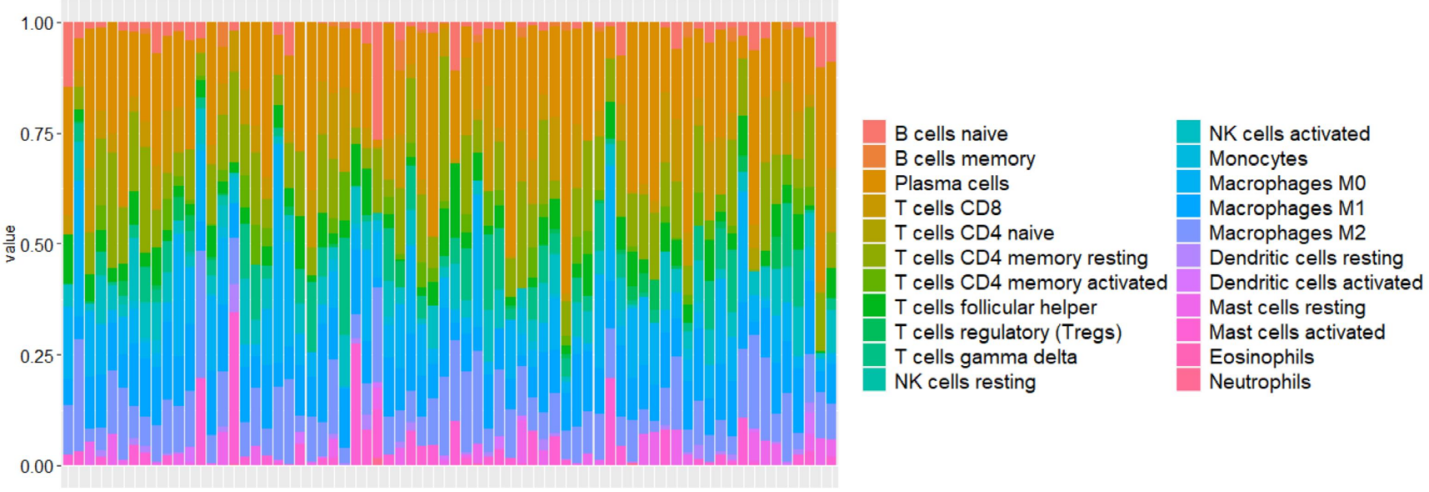

B) The proportion of 22 immune cells in low-risk patients with SCLC
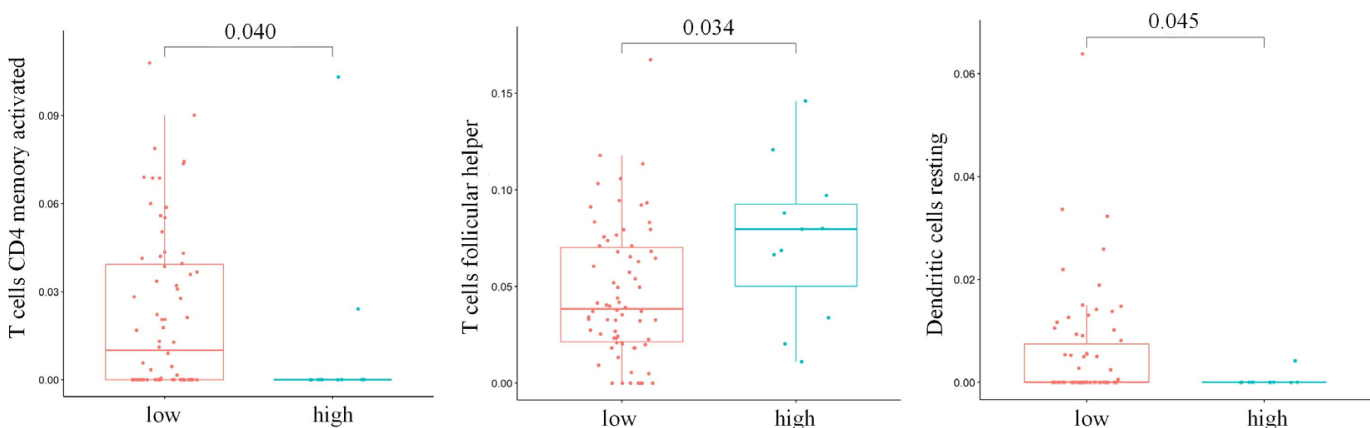

C) Differentially infiltrated immune cells in high- versus low-risk SCLC patients

Figure 4 Immune infiltration in SCLC patients with high-risk and low-risk. (A) The proportion of 22 immune cells in high-risk patients with SCLC. (B) The proportion of 22 immune cells in low-risk patients with SCLC. (C) Differentially infiltrated immune cells in high-risk and low-risk patients with SCLC, including activated memory CD4+ T cells, T follicular helper cells, and resting dendritic cells. SCLC, small cell lung cancer.

PD-L1, all 81 patients were classified into high-risk versus low-risk groups. The proportion of each immune cell differed between samples (figure 4A,B). After comparing the proportion of each immune cell among high-risk versus low-risk groups, we found SCLCpatients with high risk had a higher proportion of $\mathrm{T}$ follicular helper cells $(\mathrm{p}=0.034)$ and a lower proportion of activated memory CD4+ T cells $(p=0.040)$ and resting dendritic cells $(p=0.045)$ versus those with low risk (figure 4C). And immune cells in the high-risk group seemed to be more closely connected in comparison with those in the low-risk group (online supplemental figure 4A,B).

\section{DISCUSSION}

Immunotherapy has improved the survival of patients with SCLC, but little is known about efficacious predictive factors, which highlights the urgent need for better immune biomarkers to optimize therapeutic options. Herein, we analyzed the expression pattern of HLA class II and its connection with tumor immune microenvironment and prognosis. We detected positive HLA class II on few SCLC cells and nearly half of TILs. HLA class II on TILs was negatively correlated with lymph node metastasis and positively related to multiple immune markers and a longer RFS. Additionally, we established an HLA class II-based immune risk model that could more effectively predict the recurrence than tumor stage in SCLC. Using pathway analysis, we found signatures of transmembrane transportation, channel activity, and neuroactive ligand-receptor interaction were 
enriched in the high HLA class II expression group. And high-risk and low-risk patients with SCLC markedly differed in $\mathrm{T}$ follicular helper cells, activated memory CD4+ T cells, and resting dendritic cells. These findings indicate HLA class II plays a crucial role in tumor immune microenvironment and prognostic evaluation in patients with SCLC.

In this study, we observed that HLA class II was positively expressed on tumor cells in only $8.8 \%$ and on TILs in $44.1 \%$ of SCLC patients. The expression frequency of HLA class II on tumor cells varied among different types of cancers. Severe deficiency of HLA class II expression on tumor cells was also documented in other SCLC studies, ${ }^{21}{ }^{32}$ hepatocellular carcinomas, ${ }^{33}$ ductal breast cancers,${ }^{34}$ and acute myeloid leukemia. ${ }^{35}$ In our previous study of 72 Caucasian SCLC samples, no HLA class II was detected on tumor cells. ${ }^{21}$ The reasons for loss of HLA class II expression on tumor cells mainly included structural alterations (eg, mutations or rearrangements in HLA class II alleles) and deregulation of HLA class II antigen presentation machinery components induced by the promoter polymorphism or hypermethylation of HLA class II regulator CIITA. ${ }^{36}$ IFN- $\gamma$ played a crucial role in the regulation of HLA class II expression to ensure an immune response against tumor cells. ${ }^{36}{ }^{37}$ In vitro studies in SCLC and hepatocellular carcinomas showed the absence of HLA class II expression could not be rescued by IFN- $\gamma$ induction, ${ }^{32}{ }^{33}$ which to some extent could be considered as a form of antitumor immune escape and might partly explain their refractory features. Nonetheless, HLA class II was detectable in multiple other cancer types including NSCLC (30\%), ${ }^{21}$ primary melanomas $(50 \%-60 \%),{ }^{38}$ thyroid papillary carcinomas $(\approx 50 \%),{ }^{39}$ pancreatic cancer $(>70 \%),{ }^{40}$ and colorectal cancers $(21 \%-56 \%) .{ }^{41-43}$ As for HLA class II on TILs, our previous results revealed that HLA class II was positively expressed in only $15.3 \%$ Caucasian patients with SCLC. ${ }^{21}$ In the previous study including patients with NSCLC and SCLC, 11 (15.3\%) of 72 patients with SCLC were positive for HLA class II with a cut-off value of $80 \%$, markedly higher than $25 \%$ in this study. Given that the cut-off value was decided by the survival analysis of another NSCLC cohort with prognosis data rather than SCLC patients, the results may be incomparable. Overall, the differences of HLA class II expression level on tumor cells or TILs among a wide variety of tumors may be attributable to different research design, patient population, mechanisms of oncogenesis, molecular phenotypes, tumor origin, and various cut-off values for positivity.

We further analyzed the association of HLA class II with clinical factors. We found HLA class II expression on TILs was negatively related to lymph node metastasis, which indicated its protective function. Multiple studies have demonstrated that HLA class I was associated with lymph node metastasis in lung cancer, ${ }^{44}$ gastric cancer, ${ }^{22}{ }^{45}$ and colorectal cancer. ${ }^{46}$ However, data about the involvement of HLA class II in lymph node metastasis is limited. Ogoshi $e t$ a $t^{2}$ found HLA-DR2, HLA-DR4, and HLA-DR6 on TILs had an opposite relation to lymph node metastasis in different histological subtypes and stages of patients with gastric cancers. Paradoxical to our result, lymph node metastasis showed a positive correlation with HLA-DR4 on TILs from the blood in gastric cancer ${ }^{47}$ and HLA-DR on tumor cells in lung cancer. ${ }^{48}$ In terms of the reasons for these discrepancies, we consider different histological types or subtypes, cancer stage, and expression location of HLA class II may influence the relationship with lymph node metastasis. Our study showed SCLC patients with positive HLA class II expression on TILs had less lymph node metastasis. We speculate that better recognition of HLA class II by CD4+ T cells may lead to the elimination of tumor cells and the prevention of migration and invasion. Future study is warranted to verify it and explore potential mechanisms.

In our current research, HLA class II on TILs was coexpressed with PD-1/PD-L1 on TILs and CD3, CD4, CD8, and FOXP3 on immune cells. It indicates that HLA class II on TILs may have a close connection with multiple immune markers. On the one hand, Kitamura et $a t^{49}$ demonstrated that downregulation of HLA class II expression induced by interleukin-6/STAT3 signaling activation hampered dendritic cells activating effector $\mathrm{T}$ cells in colorectal cancer. The impairment of HLA class II on TILs could account for the immunosuppression in NSCLC..$^{50}$ On the other hand, considerable evidence supported T cells expressing HLA class II could function as APCs, also called T-APCs, and antigen presentation mediated by T-APCs could induce apoptosis or clonal anergy in activated $\mathrm{T}$ cells or cytotoxicity in resting $\mathrm{T}$ cells. ${ }^{20}$ Correspondingly, HLA class II-specific monoclonal antibodies were developed to treat hematological malignancy. ${ }^{51}$ Additionally, Costantini $e t a l^{18}$ found HLA-DR mediated signaling increased the expression of adhesion receptors and PD-L1 and the activation of the JAK/STAT3 pathway, and promoted progression, migration, and invasion of melanoma cells. Taken together, HLA class II played an important role in tumor immune microenvironment, oncogenesis, and tumor progression. But it remains to be elucidated whether this association reflects the attraction of TILs by HLA class II or the upregulation of HLA class II-induced by IFN- $\gamma$ secreted by TILs.

We also found HLA class II expressed on tumor cells did not correlate with clinical and other immune factors except for PD-1 on TILs. Similar results of insignificant correlation between HLA class II on tumor cells with CD4/CD8+ T cells was observed in intrahepatic cholangiocarcinoma. ${ }^{52}$ However, a significant association was observed between HLA class II expression and multiple immune molecules and cells, including PD-1/ PD-L1 and CD8+ TILs in melanoma cells, ${ }^{18}{ }_{53}$ CD4+ TILs in lymphomas and colorectal cancers, ${ }^{54}{ }^{55} \mathrm{CD} 3+$ TILs in mismatch repair-deficient cancers,${ }^{56}$ and CD68+ and $\mathrm{CD} 163+$ tumor-associated macrophages in oropharyngeal cancer. ${ }^{57}$ As for SCLC, HLA class II on tumor cells had a limited association with immune markers, which might be ascribed to the extremely low level of HLA class II expression on tumor cells in SCLC in comparison with other types of cancers. Future larger sample-size clinical researches or fundamental investigation may further ascertain their association and potential mechanisms. 
We observed RFS had no significant difference in SCLC patients with different expression status of HLA class II on tumor cells, and its rare expression limited further analysis. Nonetheless, RFS was significantly longer in SCLC patients positive for HLA class II on TILs as opposed to those negative, in accordance with our previous study. ${ }^{21}$ This favorable prognostic connection is not unique to SCLC, as it has recently been reported in a variety of solid and hematological tumors. ${ }^{39}{ }^{57-60}$ In lymphoma and melanoma, HLA class II positivity on tumor cells was not only a favorable prognostic predictor but also an efficacious predictor of PD-1 blockade, thus producing the hypothesis of HLA class II-limited, CD4+ TILs-mediated action mechanism of PD-1 blockade. ${ }^{58} 6061$ Additionally, the association of HLA class II with immune factors may partly account for its prognostic implication. Despite a lot of evidence supporting the favorable prognostic implications of positive HLA class II, other studies showed a discrepant correlation. HLA class II antigen was related to a poor prognosis in hepatocellular carcinoma and osteosarcomas. ${ }^{62} 63$ The inverse results may be induced by different oncogenesis mechanisms and heterogeneity of HLA class II subtypes. Overall, HLA class II has a definite link to RFS in SCLC, and its prognostic significance may be partly attributed to a close immune interaction.

Considering the close connection between HLA class II and immune markers and prognosis, we performed LASSO regression analysis among multiple immune variables to further analyze the influential factors of RFS in SCLC. And then we determined an HLA class II-based optimal risk model that categorized patients into highrisk and low-risk groups. High-risk patients with SCLC had a poorer prognosis, which was further confirmed in the public dataset. To our knowledge, it is the first time that we build an HLA class II-based immune risk model for prognostic evaluation in patients with SCLC, and it even has better predictive power than tumor stage.

In the final immune risk model, PD-L1, CD4, and FOXP3 were protective factors of RFS in patients with SCLC. PD-L1 on tumor cells or TILs was higher among early-stage SCLC compared with metastatic patients, ${ }^{64}$ and multiple authors demonstrated an association with better outcome in SCLC. ${ }^{64-68}$ However, limited studies reported contrary results. ${ }^{69}$ Different antibodies, cut-off values, and study design may cause the discrepancy. In our study, CD4+ TILs were closely associated with HLA class II and better prognosis in SCLC. CD4+ TILs recognition of HLA class II-restricted antigen could activate antitumor immunity. ${ }^{11} \mathrm{CD} 4+\mathrm{T}$ cells activation promoted differentiation into effector $\mathrm{T}$ cells and regulatory $\mathrm{T}$ (Treg) cells. The number of effector T cells in LS-SCLC was significantly more than that in ES-SCLC, and the high ratio of effector $\mathrm{T}$ cells to Treg cells predicted a long-term survival. ${ }^{71}$ FOXP3 is a specific biomarker of Treg cells, and its role in cancers is conflicting. ${ }^{72}$ Especially for SCLC, FOXP3+TILs presented inconsistent associations with prognosis. In a retrospective SCLC study, FOXP3+TILs were an independent positive prognostic factor in patients with nonmetastatic SCLC at stage I-III, but not in metastatic patients. ${ }^{64}$ Our study also exhibited a positive association of FOXP3 with prognosis among patients with surgically resected SCLC at stage I-III. However, Kasahara et $a l^{73}$ found an insignificant correlation between FOXP3 and OS in patients with both LS-SCLC and ES-SCLC. The authors determined the cut-off value of FOXP3 based on survival analysis, but no specific value was provided. As for patients with metastatic SCLC, a similar insignificant association with survival was observed in another study that performed FOXP3+TILs IHC staining on brain metastasis specimens of patients with SCLC. ${ }^{65}$ Wang $e t a l^{74}$ included 65 SCLC patients without stage information and demonstrated patients with a higher ratio of FOXP3/CD45 cells (measured as Tregs) had a worse median OS (120 vs 410 days). Considering its short OS, it may mainly consist of metastatic patients. The heterogeneous association of FOXP3 with prognosis in SCLC may be partly attributable to non-uniform cut-off values, tumor stage, and detection locations (primary vs metastatic). Additionally, FOXP3positive $\mathrm{T}$ cells were constituted by three subpopulations: CD45RA(+)FOXP3(lo), CD45RA(-)FOXP3(hi), and CD45RA(-)FOXP3(lo) ${ }^{75}$ The former two were suppressive, and the third was non-suppressive $\mathrm{T}$ cells characterized by FOXP3 instability and inflammatory cytokines secretion. An abundance of inflammatory CD45RA(-) FOXP3(lo) T cells was correlated with a better prognosis in colorectal cancer. ${ }^{76}$ Therefore, the distinct predominant subpopulations of FOXP3+TILs during the dynamic process of tumor formation and progression may account for the different influences on prognosis in SCLC.

Furthermore, with bioinformatics methods, we investigated the implications of HLA class II and the immune risk model composed of HLA class II, PD-L1, CD4, and FOXP3 in immune infiltration and prognosis. After observing those SCLC patients with high HLA class II expression had a better prognosis versus low-expressed patients, we first performed GO and KEGG enrichment analyses to explore the potential biological pathways associated with HLA class II. Enriched pathways in the high-expression group involved transmembrane transport, channel activity, and ligand-receptor interaction. It indicates that HLA class II expression is associated with signaling transduction, as demonstrated in previous studies where ligation of HLA class II could transduce multiple intracellular signals such as protein kinase $\mathrm{C}$ membrane translocation and promote calcium influx..$^{20} 77$ Then we did CIBERSORT analysis to delineate the immune infiltration landscape among high-risk and low-risk patients with SCLC. We found high-risk patients seemed to possess a closer interaction with immune cells and had significantly lower proportions of activated memory $\mathrm{CD} 4+\mathrm{T}$ cells and resting dendritic cells versus those with low-risk. These differentially infiltrated immune cells correlated closely with antigen presentation and immune response. Collectively, we demonstrated a close relationship between HLA class II molecules, tumor immune microenvironment, and prognosis. Multiple studies mentioned that, except for the well-established role of tumor neoantigen-specific 
CD8+ $\mathrm{T}$ cells in antitumor immunity, HLA class II presenting neoantigens to CD4+ T cells also exerted a crucial function in tumor eradication and response to immunotherapy. ${ }^{5878} 79$ Despite that, some cancer patients responded to immunotherapy even without detectable HLA class II ${ }^{58}$ indicating that the mechanisms of HLA class II in immune regulation and antitumor immunity remain to be elaborated.

Finally, we acknowledge several limitations in our research. First of all, lack of representativeness may present as this study is single-center and retrospective. Second, given that there are no clear and standard cut-off values for HLA class II and multiple immune indicators, we determined the optimal cut-off values for immune markers by survival analysis. The repeatability and clinical utility of the optimal cut-off values need more studies to verify. Third, we established an immune risk model after cross-validation in the LASSO regression. Further validation and refinement are warranted in the future studies. Finally, not enough long follow-up duration restrains from comparing OS, but RFS already showed a sufficiently significant difference.

\section{CONCLUSION}

In this study, we detected positive HLA class II on few SCLC cells and nearly half of TILs. And we found positivity of HLA class II on TILs was negatively associated with lymph node metastasis and positively related to multiple immune markers and a better RFS. Furthermore, we established an HLA class II-based immune risk model, which can not only more effectively predict the recurrence than tumor stage but also identify immune infiltration heterogeneity in high-risk and low-risk patients with SCLC. Taken together, these findings indicate that HLA class II plays a crucial role in tumor immune microenvironment and prognostic evaluation in SCLC patients. Future studies are warranted to reveal the mechanism behind it.

\section{Author affiliations \\ ${ }^{1}$ Department of Medical Oncology, Shanghai Pulmonary Hospital, Tongji University Medical School Cancer Institute, School of Medicine, Tongji University, Shanghai 200092, China \\ ${ }^{2}$ Tongji University, No 1239 Siping Road, Shanghai 200433, China \\ ${ }^{3}$ Department of Oncology, the Second Xiangya Hospital, Central South University, Changsha 410011, China \\ ${ }^{4}$ Department of Pathology, Shanghai Pulmonary Hospital, Tongji University Medical School Cancer Institute, School of Medicine, Tongji University, Shanghai 200092, China \\ ${ }^{5}$ Anhui No.2 Provincial People's Hospital, Hefei, China}

Contributors YH, LZhao, and PC designed the study. CZ and YH designed the whole project and supervised all experiments. WZ, CS, and CW performed the experiments; PC, HW, LZhang, JZ, JY, SZ, and WL collected the data; LZhao analyzed the data, drafted, and revised the manuscript. PC did bioinformatics analysis. All authors approved the final manuscript.

Funding This study was supported in part by a grant of National Natural Science Foundation of China (81802255), Clinical research project of Shanghai Pulmonary Hospital (FKLY20010), Young Talents in Shanghai (2019 QNBJ), 'Dream Tutor' Outstanding Young Talents Program (fkyq1901), Clinical research project of Shanghai Pulmonary Hospital (FKLY20001), Respiratory medicine, a key clinical specialty construction project in Shanghai, Promotion and application of multidisciplinary collaboration system for pulmonary non infectious diseases, Clinical Research Project of Shanghai Pulmonary Hospital (fk18005), Key Discipline in 2019 (oncology), Project of Shanghai Municipal Science and Technology Commission (Project of Municipal Science and Technology Commission), Scientific research project of Shanghai Pulmonary Hospital (fkcx1903), Shanghai Municipal Commission of Health and Family Planning (2017YQ050), Innovation Training Project of SITP of Tongji University, and key projects of leading talent (19411950300), Youth project of hospital management research fund of Shanghai Hospital Association (Q1902037).

Competing interests None declared.

Patient consent for publication Not required.

Ethics approval This study was approved by the Ethics Committee of Shanghai Pulmonary Hospital (ethical number K20-022). All patients signed consents form to participate in this study.

Provenance and peer review Not commissioned; externally peer reviewed.

Data availability statement Data are available upon reasonable request. All data relevant to the study are included in the article or uploaded as supplementary information. Data are available upon reasonable request. All data relevant to this study are included in the article or online supplemental materials.

Supplemental material This content has been supplied by the author(s). It has not been vetted by BMJ Publishing Group Limited (BMJ) and may not have been peer-reviewed. Any opinions or recommendations discussed are solely those of the author(s) and are not endorsed by BMJ. BMJ disclaims all liability and responsibility arising from any reliance placed on the content. Where the content includes any translated material, BMJ does not warrant the accuracy and reliability of the translations (including but not limited to local regulations, clinical guidelines, terminology, drug names and drug dosages), and is not responsible for any error and/or omissions arising from translation and adaptation or otherwise.

Open access This is an open access article distributed in accordance with the Creative Commons Attribution 4.0 Unported (CC BY 4.0) license, which permits others to copy, redistribute, remix, transform and build upon this work for any purpose, provided the original work is properly cited, a link to the licence is given, and indication of whether changes were made. See https://creativecommons.org/ licenses/by/4.0/.

\section{ORCID iDs}

Peixin Chen http://orcid.org/0000-0002-4192-8396

Lishu Zhao http://orcid.org/0000-0003-4728-9107

Yayi He http://orcid.org/0000-0002-2820-9119

Caicun Zhou http://orcid.org/0000-0002-7095-0381

\section{REFERENCES}

1 Siegel RL, Miller KD, Jemal A. Cancer statistics, 2020. CA Cancer J Clin 2020;70:7-30.

2 Lum C, Alamgeer M. Technological and therapeutic advances in advanced small cell lung cancer. Cancers 2019;11:1570.

3 Koinis F, Kotsakis A, Georgoulias V. Small cell lung cancer (SCLC): no treatment advances in recent years. Transl Lung Cancer Res 2016;5:39-50.

4 Wang S, Zimmermann S, Parikh K, et al. Current diagnosis and management of small-cell lung cancer. Mayo Clin Proc 2019;94:1599-622.

5 Rudin CM, Ismaila N, Hann CL, et al. Treatment of small-cell lung cancer: American Society of clinical oncology endorsement of the American College of chest physicians guideline. J Clin Oncol 2015;33:4106-11.

6 O'Donnell JS, Teng MWL, Smyth MJ. Cancer immunoediting and resistance to T cell-based immunotherapy. Nat Rev Clin Oncol 2019;16:151-67.

7 Horn L, Mansfield AS, Szczęsna A, et al. First-Line Atezolizumab plus chemotherapy in extensive-stage small-cell lung cancer. $N$ Engl $J$ Med 2018;379:2220-9.

8 Yang S, Zhang Z, Wang Q. Emerging therapies for small cell lung cancer. J Hematol Oncol 2019;12:47.

9 Paz-Ares L, Dvorkin M, Chen Y, et al. Durvalumab plus platinumetoposide versus platinum-etoposide in first-line treatment of extensive-stage small-cell lung cancer (Caspian): a randomised, controlled, open-label, phase 3 trial. Lancet 2019;394:1929-39. 
10 Gelsomino F, Lamberti G, Parisi C, et al. The evolving landscape of immunotherapy in small-cell lung cancer: a focus on predictive biomarkers. Cancer Treat Rev 2019;79:101887.

11 Marty Pyke R, Thompson WK, Salem RM, et al. Evolutionary pressure against $\mathrm{MHC}$ class II binding cancer mutations. Cell 2018;175:416-28.

12 Dendrou CA, Petersen J, Rossjohn J, et al. Hla variation and disease. Nat Rev Immunol 2018;18:325-39.

13 Xu Y, Wang L, Li W, et al. Killer immunoglobulin-like receptors/human leukocyte antigen class-I, a crucial immune pathway in cancer. Ann Transl Med 2020;8:244.

14 Reith W, LeibundGut-Landmann S, Waldburger J-M. Regulation of MHC class II gene expression by the class II transactivator. Nat Rev Immunol 2005;5:793-806.

15 Seliger B, Ruiz-Cabello F, Garrido F. Ifn inducibility of major histocompatibility antigens in tumors. Adv Cancer Res 2008;101:249-76.

16 Giroux M, Schmidt M, Descoteaux A. Ifn-Gamma-Induced MHC class II expression: transactivation of class II transactivator promoter IV by IFN regulatory factor- 1 is regulated by protein kinase $\mathrm{C}$-alpha. $\mathrm{J}$ Immunol 2003;171:4187-94.

17 van den Hoorn T, Paul P, Jongsma MLM, et al. Routes to manipulate MHC class II antigen presentation. Curr Opin Immunol 2011;23:88-95.

18 Costantini F, Barbieri G. The HLA-DR mediated signalling increases the migration and invasion of melanoma cells, the expression and lipid raft recruitment of adhesion receptors, PD-L1 and signal transduction proteins. Cell Signal 2017;36:189-203.

19 Zagzag D, Salnikow K, Chiriboga L, et al. Downregulation of major histocompatibility complex antigens in invading glioma cells: stealth invasion of the brain. Lab Invest 2005;85:328-41.

20 Holling TM, Schooten E, van Den Elsen PJ. Function and regulation of MHC class II molecules in T-lymphocytes: of mice and men. Hum Immunol 2004;65:282-90.

$21 \mathrm{He}$ Y, Rozeboom L, Rivard CJ, et al. Mhc class II expression in lung cancer. Lung Cancer 2017;112:75-80.

22 Ogoshi K, Tajima T, Mitomi T, et al. Hla-A2 antigen status predicts metastasis and response to immunotherapy in gastric cancer. Cancer Immunol Immunother 1997;45:53-9.

23 Detterbeck FC, Boffa DJ, Kim AW, et al. The eighth edition lung cancer stage classification. Chest 2017:151:193-203.

24 Nicholson AG, Chansky K, Crowley J, et al. The Internationa association for the study of lung cancer lung cancer staging project: proposals for the revision of the clinical and pathologic staging of small cell lung cancer in the forthcoming eighth edition of the TNM classification for lung cancer. J Thorac Oncol 2016;11:300-11.

$25 \mathrm{He} \mathrm{Y,} \mathrm{Yu} \mathrm{H,} \mathrm{Rozeboom} \mathrm{L,} \mathrm{et} \mathrm{al.} \mathrm{LAG-3} \mathrm{protein} \mathrm{expression} \mathrm{in} \mathrm{non-}$ small cell lung cancer and its relationship with PD-1/PD-L1 and tumor-infiltrating lymphocytes. J Thorac Oncol 2017;12:814-23.

$26 \mathrm{He}$ Y, Rozeboom L, Rivard CJ, et al. Pd-1, PD-L1 protein expression in non-small cell lung cancer and their relationship with tumorinfiltrating lymphocytes. Med Sci Monit 2017;23:1208-16.

27 Camp RL, Dolled-Filhart M, Rimm DL. X-tile: a new bio-informatics tool for biomarker assessment and outcome-based cut-point optimization. Clin Cancer Res 2004;10:7252-9.

28 Sun C, Zhang L, Zhang W, et al. Expression of PD-1 and PD-L1 on tumor-infiltrating lymphocytes predicts prognosis in patients with small-cell lung cancer. Onco Targets Ther 2020;13:6475-83.

29 Subramanian A, Tamayo P, Mootha VK, et al. Gene set enrichment analysis: a knowledge-based approach for interpreting genome-wide expression profiles. Proc Natl Acad Sci U S A 2005;102:15545-50.

30 Newman AM, Liu CL, Green MR, et al. Robust enumeration of cell subsets from tissue expression profiles. Nat Methods 2015;12:453-7.

31 George J, Lim JS, Jang SJ, et al. Comprehensive genomic profiles of small cell lung cancer. Nature 2015;524:47-53.

32 Yazawa T, Kamma H, Fujiwara M, et al. Lack of class II transactivator causes severe deficiency of HLA-DR expression in small cell lung cancer. J Pathol 1999;187:191-9.

33 Ramia E, Chiaravalli AM, Bou Nasser Eddine F, et al. CIITArelated block of HLA class II expression, upregulation of HLA class $\mathrm{I}$, and heterogeneous expression of immune checkpoints in hepatocarcinomas: implications for new therapeutic approaches. Oncoimmunology 2019;8:1548243.

34 Concha A, Ruiz-Cabello F, Cabrera T, et al. Different patterns of HLA-DR antigen expression in normal epithelium, hyperplastic and neoplastic malignant lesions of the breast. Eur J Immunogenet 1995:22:299-310.

35 Toffalori C, Zito L, Gambacorta V, et al. Immune signature drives leukemia escape and relapse after hematopoietic cell transplantation. Nat Med 2019;25:603-11.
36 Seliger B, Kloor M, Ferrone S. Hla class II antigen-processing pathway in tumors: molecular defects and clinical relevance. Oncoimmunology 2017;6:e1171447.

37 Newey A, Griffiths B, Michaux J, et al. Immunopeptidomics of colorectal cancer organoids reveals a sparse HLA class I neoantigen landscape and no increase in neoantigens with interferon or MEKinhibitor treatment. J Immunother Cancer 2019;7:309.

38 Taramelli D, Fossati G, Mazzocchi A, et al. Classes I and II HLA and melanoma-associated antigen expression and modulation on melanoma cells isolated from primary and metastatic lesions. Cancer Res 1986;46:433-9.

39 Jo YS, Lee JC, Li S, et al. Significance of the expression of major histocompatibility complex class II antigen, HLA-DR and -DQ, with recurrence of papillary thyroid cancer. Int J Cancer 2008;122:785-90.

40 Gaida MM, Welsch T, Herpel E, et al. Mhc class II expression in pancreatic tumors: a link to intratumoral inflammation. Virchows Arch 2012;460:47-60.

41 Wolkersdörfer T, Füssel M, Kiesslich T, et al. Mhc class II genotypeand $\mathrm{MHC}$ class I and II phenotype-related parameters in sporadic colorectal cancer. Oncol Rep 2011;26:1165-71.

42 McDougall CJ, Ngoi SS, Goldman IS, et al. Reduced expression of HLA class I and II antigens in colon cancer. Cancer Res 1990;50:8023-7.

43 Gutierrez J, López-Nevot MA, Cabrera T, et al. Class I and II HLA antigen distribution in normal mucosa, adenoma and colon carcinoma: relation with malignancy and invasiveness. Exp Clin Immunogenet 1987;4:144-52.

44 Bulut I, Meral M, Kaynar H, et al. Analysis of HLA class I and II alleles regarding to lymph node and distant metastasis in patients with nonsmall cell lung cancer. Lung Cancer 2009;66:231-6.

45 Ueda Y, Ishikawa K, Shiraishi N, et al. Clinical significance of HLA class I heavy chain expression in patients with gastric cancer. J Surg Oncol 2008:97:451-5.

46 Ye S-R, Yang H, Li K, et al. Human leukocyte antigen G expression: as a significant prognostic indicator for patients with colorectal cancer. Mod Pathol 2007;20:375-83.

47 Ogoshi K, Tajima T, Mitomi T, et al. Hla antigens are candidate markers for prediction of lymph node metastasis in gastric cancer. Clin Exp Metastasis 1996;14:277-81.

48 Nakamura H, Kawasaki N, Hagiwara M, et al. Cellular immunologic parameters related to age, gender, and stage in lung cancer patients. Lung Cancer 2000;28:139-45.

49 Kitamura H, Ohno Y, Toyoshima Y, et al. Interleukin-6/Stat3 signaling as a promising target to improve the efficacy of cancer immunotherapy. Cancer Sci 2017;108:1947-52.

50 Foukas PG, Tsilivakos V, Zacharatos P, et al. Expression of HLA-DR is reduced in tumor infiltrating immune cells (TIICs) and regional lymph nodes of non-small-cell lung carcinomas. A putative mechanism of tumor-induced immunosuppression? Anticancer Res 2001;21:2609-15.

51 Nagy ZA, Hubner B, Löhning C, et al. Fully human, HLA-DR-specific monoclonal antibodies efficiently induce programmed death of malignant lymphoid cells. Nat Med 2002;8:801-7.

52 Sabbatino F, Villani V, Yearley JH, et al. Pd-L1 and HLA class I antigen expression and clinical course of the disease in intrahepatic cholangiocarcinoma. Clin Cancer Res 2016;22:470-8.

53 Ladányi A, Papp E, Mohos A, et al. Role of the anatomic site in the association of HLA class I antigen expression level in metastases with clinical response to ipilimumab therapy in patients with melanoma. J Immunother Cancer 2020;8:e000209.

54 Ansell SM, Stenson M, Habermann TM, et al. Cd4+ T-cell immune response to large B-cell non-Hodgkin's lymphoma predicts patient outcome. J Clin Oncol 2001;19:720-6.

55 Surmann E-M, Voigt AY, Michel S, et al. Association of high CD4positive T cell infiltration with mutations in HLA class II-regulatory genes in microsatellite-unstable colorectal cancer. Cancer Immunol Immunother 2015;64:357-66.

56 Janikovits J, Müller M, Krzykalla J, et al. High numbers of PDCD1 (PD-1)-positive T cells and B2M mutations in microsatellite-unstable colorectal cancer. Oncoimmunology 2018;7:e1390640.

57 Cioni B, Jordanova ES, Hooijberg E, et al. Hla class II expression on tumor cells and low numbers of tumor-associated macrophages predict clinical outcome in oropharyngeal cancer. Head Neck 2019;41:463-78

58 Roemer MGM, Redd RA, Cader FZ, et al. Major histocompatibility complex class II and programmed death ligand 1 expression predict outcome after programmed death 1 blockade in classic Hodgkin lymphoma. J Clin Oncol 2018;36:942-50.

59 Sconocchia G, Eppenberger-Castori S, Zlobec I, et al. Hla class II antigen expression in colorectal carcinoma tumors as a favorable prognostic marker. Neoplasia 2014;16:31-42. 
60 Takeuchi M, Miyoshi H, Asano N, et al. Human leukocyte antigen class II expression is a good prognostic factor in adult T-cell leukemia/lymphoma. Haematologica 2019;104:1626-32.

61 Johnson DB, Estrada MV, Salgado R, et al. Melanoma-Specific MHC-II expression represents a tumour-autonomous phenotype and predicts response to anti-PD-1/PD-L1 therapy. Nat Commun 2016;7:10582.

62 Zhou Q, Xu J, Zhao J, et al. Downregulation of CD99 and upregulation of human leukocyte antigen class II promote tumor aggravation and poor survival in patients with osteosarcomas. Onco Targets Ther 2014;7:477-84

63 Matoba K, lizuka N, Gondo T, et al. Tumor HLA-DR expression linked to early intrahepatic recurrence of hepatocellular carcinoma. Int $J$ Cancer 2005;115:231-40.

64 Bonanno L, Pavan A, Dieci MV, et al. The role of immune microenvironment in small-cell lung cancer: distribution of PD-L1 expression and prognostic role of FOXP3-positive tumour infiltrating lymphocytes. Eur J Cancer 2018;101:191-200.

65 Berghoff AS, Ricken G, Wilhelm D, et al. Tumor infiltrating lymphocytes and PD-L1 expression in brain metastases of small cell lung cancer (SCLC). J Neurooncol 2016;130:19-29.

66 Ishii H, Azuma K, Kawahara A, et al. Significance of programmed cell death-ligand 1 expression and its association with survival in patients with small cell lung cancer. $J$ Thorac Oncol 2015;10:426-30.

$67 \mathrm{Yu} \mathrm{H}$, Batenchuk C, Badzio A, et al. Pd-L1 expression by two complementary diagnostic assays and mRNA in situ hybridization in small cell lung cancer. J Thorac Oncol 2017;12:110-20.

68 Toyokawa G, Takada K, Haratake N, et al. Favorable disease-free survival associated with programmed death ligand 1 expression in patients with surgically resected small-cell lung cancer. Anticancer Res 2016;36:4329-36.

69 Zhao X, Kallakury B, Chahine JJ, et al. Surgical resection of SCLC: prognostic factors and the tumor microenvironment. $J$ Thorac Oncol 2019;14:914-23.
70 Fu X, Liu Z, Xiang L, et al. Pd-L1 predicts poor prognosis in surgically resected limited stage small-cell lung cancer. Cancer Manag Res 2020;12:10939-48.

71 Koyama K, Kagamu H, Miura S, et al. Reciprocal CD4+ T-cell balance of effector CD62Llow CD4+ and CD62LhighCD25+ CD4+ regulatory $\mathrm{T}$ cells in small cell lung cancer reflects disease stage. Clin Cancer Res 2008;14:6770-9.

72 Yang S, Liu Y, Li M-Y, et al. Foxp3 promotes tumor growth and metastasis by activating Wnt/ $\beta$-catenin signaling pathway and EMT in non-small cell lung cancer. Mol Cancer 2017;16:124.

73 Kasahara N, Kaira K, Yamaguchi K, et al. Fluorodeoxyglucose uptake is associated with low tumor-infiltrating lymphocyte levels in patients with small cell lung cancer. Lung Cancer 2019;134:180-6.

74 Wang W, Hodkinson P, McLaren F, et al. Small cell lung cancer tumour cells induce regulatory $T$ lymphocytes, and patient survival correlates negatively with Foxp3+ cells in tumour infiltrate. Int $J$ Cancer 2012;131:E928-37.

75 Miyara M, Yoshioka Y, Kitoh A, et al. Functional delineation and differentiation dynamics of human CD4+ T cells expressing the FOXP3 transcription factor. Immunity 2009;30:899-911.

76 Saito T, Nishikawa H, Wada H, et al. Two FOXP3(+)CD4(+) T cell subpopulations distinctly control the prognosis of colorectal cancers. Nat Med 2016;22:679-84.

77 Di Rosa F, D'Oro U, Ruggiero G, et al. HLA class II molecules transduce accessory signals affecting the CD3 but not the interleukin-2 activation pathway in T blasts. Hum Immunol 1993;38:251-60.

78 Alspach E, Lussier DM, Miceli AP, et al. MHC-II neoantigens shape tumour immunity and response to immunotherapy. Nature 2019;574:696-701.

79 Thibodeau J, Bourgeois-Daigneault M-C, Lapointe R. Targeting the $\mathrm{MHC}$ class II antigen presentation pathway in cancer immunotherapy. Oncoimmunology 2012;1:908-16. 